\title{
Identification of pluripotent cells in bovine uterus: in situ and in vitro studies
}

\author{
Martyna Łupicka ${ }^{1}$, Gabriel Bodek ${ }^{2}$, Nahum Shpigel ${ }^{3}$, Ehud Elnekave ${ }^{3}$ and Anna J Korzekwa ${ }^{1}$ \\ ${ }^{1}$ Department of Reproductive Immunology and Pathology and ${ }^{2}$ In Vitro and Cell Biotechnology Laboratory, Institute of \\ Animal Reproduction and Food Research, Polish Academy of Sciences, 10-748 Olsztyn, Poland and ${ }^{3}$ Koret School of \\ Veterinary Medicine, The University of Jerusalem, Rehovot 76100, Israel
}

Correspondence should be addressed to A J Korzekwa; Email: a.korzekwa@pan.olsztyn.pl

\begin{abstract}
The aim of this study was to identify uterine pluripotent cells both in bovine uterine tissues as well in epithelial, stromal, and myometrial uterine cell populations. Moreover, the relationship of pluripotent markers expression with age and the uterine horn side was considered. Uterine tissue was collected from ipsilateral and contralateral horns (days 8-10 of the estrous cycle). Immunohistostaining for C-KIT, $O C T 3 / 4, N A N O G$, and SOX2 in uterine tissue was determined. mRNA expression of C-KIT, OCT3/4, NANOG and SOX2 was evaluated in uterine tissue relative to the age of the cow and uterine horn side. Gene and protein expression of these markers in the uterine luminal epithelial, stromal, and myometrial cells was evaluated by real-time PCR and western blotting respectively. The expression of pluripotent cell markers $O C T 3 / 4, N A N O G$, and SOX2 was identified by flow cytometry assay in epithelial, stromal, and myometrial cells. Multilineage differentiation of the bovine uterine cells was performed. mRNA expression of OCT3/4, NANOG, and SOX2 in uterine tissue was higher in the ipsilateral horn than in the contralateral horn. Flow cytometry assay revealed positive fluorescence for OCT3/4, NANOG, and SOX2 in all uterine cell types. Results showed the age-dependent expression of pluripotent markers in uterine tissue. Beside, the different expression of pluripotent cells in each horn of uterus suggests the influence of ovarian hormones on these characteristics. The highest mRNA and protein expression for pluripotent markers was observed in stromal cells among uterine cells, which indicates this population of cells as the main site of pluripotent cells in the cow uterus.

Reproduction (2015) 149 317-327
\end{abstract}

\section{Introduction}

Stem cells are undifferentiated, primary cells that are able to self-renew by producing identical daughter cells, or to produce daughter cells of multiple cell lineages, that are more specialized than the parent cells (Gargett 2004). There are two main kinds of stem cells: embryonic stem cells (ESc) and adult stem cells (persisting in adult organs). ESc are derived from the inner cell mass of the blastocyst and are pluripotent, which means that they possess the ability to differentiate into cells of all three germ layers: ectoderm, mesoderm, and endoderm (Du \& Taylor 2009). The adult stem cells reside in many organs and tissues in structures called niches. These niches provide the stem cells with a proper microenvironment and signal them to divide into daughter cells (Figueira et al. 2011). The adult stem cells, unlike pluripotent ESc, are multipotent and can produce a limited range of cell lineages depending on their location. However, recent studies have proven that the plasticity of adult stem cells is more extensive. These cells are mainly involved in tissue regeneration and growth (Du \& Taylor 2009). Changes that occur in the endometrium during reproductive cycles require remarkable proliferation capacity, thus stem cells may be involved in tissue growth, remodeling, and regenerative processes (Gargett 2004, Gargett et al. 2012).

During reproductive cycles and pregnancy, under the influence of ovarian hormones, the uterus undergoes intensive changes that are likely supported by uterine stem cells in women and mice (Figueira et al. 2011, $\mathrm{Xu}$ et al. 2011). The estrogen levels are elevated during the proliferative/follicular phase of the estrous cycle and cyclically promote proliferation of luminal and glandular epithelial cells in the endometrium. It has been suggested that the regeneration and growth of new glands and stromal morphology elements in the endometrium are the product of stem cell populations (Gargett 2004, Teixeira et al. 2008). In cow proper cyclicity and endometrial functions are extremely important for breeding reproductive status of the animal. The ovarian hormone-dependent morphogenesis of endometrial glands is probably the result of stem cells proliferation and the key aspect for successful implantation of bovine embryo (Spencer et al. 2004). Therefore 
our study concerns the identification of stem cells in the bovine uterus.

Cells derived from human endometrial tissue have clonogenic activity and are able to give rise to mesenchymal lineages in vitro, such as adipocytes, smooth muscle cells, chondrocytes, and osteoblasts, which suggest that these cells constitute the population of mesenchymal stem cells (MSc; Schwab \& Gargett 2007). In women, the basal lining of the endometrium, which is known to be responsible for regeneration of cyclically shedded endometrial functional layer, is the most likely place in which endometrial stem cells reside (Maruyama et al. 2010). Recent studies have also pointed out the presence of stem cells in the myometrial layer of the human uterus as well, where they contribute to uterine remodeling, especially during pregnancy (Ono et al. 2012, Maruyama et al. 2013). Abnormalities in uterine stem cell functions are potentially associated with the development of gynecological disorders such as adenomyosis, uterine cancers, or endometriosis in women. These diseases are connected with abnormal proliferation of uterine cells, which may occur when stem cells are exposed to pathological hormonal stimuli (Gargett et al. 2012).

Several markers for stem cells in human uterus have been identified; however, these markers are not highly specific for endometrial stem cells (Schwab et al. 2008). In the endometrium, the most commonly mentioned markers, indicating stem cell properties are cell membrane antigen $C-K I T$, also known as $C D 117$, and intracellular transcription factor OCT3/4. The C-KIT marker is usually associated with hematopoietic stem cells and its expression may suggest a medullary origin of these stem cells (Cho et al. 2004, Schwab et al. 2008). Moreover, identification of two other nuclear transcription factors, NANOG and SOX2, allows for determining the presence of stem cells in the uterus. These markers, which are characteristic for pluripotent cells, are expressed in ESc (Calloni et al. 2013) and, as recent studies have shown, also in MSc, settled in reproductive organs (Izadpanah et al. 2005, Lee et al. 2013, Stimpfel et al. 2013). Therefore, we selected OCT3/4, NANOG, and SOX2 as the markers of non-differentiated state and pluripotency/multipotency of cells residing in the bovine uterus. We have also determined the expression of C-KIT as the marker of multipotent cells that may migrate into uterine tissues from bone marrow.

In this study, we hypothesized that pluripotent cells are present in the bovine uterus, in different uterine cell populations, and their functions are age and uterine horn side dependent, thus we i) localized the pluripotent markers in bovine uterine tissue; ii) studied the $C-K I T$, OCT3/4, NANOG, and SOX2 mRNA expression in uterine tissues, relative to the age of cows and uterine horn side; iii) determined C-KIT, OCT3/4, NANOG, and SOX2 expression at mRNA and protein level in uterine cells; iv) identified stem cell markers, OCT3/4, NANOG, and SOX2, in bovine uterine cell populations derived from different uterine layers by flow cytometry; v) confirmed pluripotency/multipotency of the bovine uterine cells by multilineage differentiation.

\section{Materials and methods}

A total of 32 healthy cows were used for post-mortem collection of uteri. Of those, 24 Israeli Holstein Fresian Black and White cows uteri were used for determination of mRNA and protein localization in experiments 1 and 2, and other uteri $(n=8)$ from Holstein/Polish Black and White cows $(75 / 25 \%$ respectively) for in vitro studies in experiments 3,4 , and 5 .

Before slaughter, for each cow, information about the age and the number of lactations was recorded and absence of previous metritis was confirmed. The reasons for culling animals from the herd were economic aspects and renewal of the herd in the case of older cows.

For experiments 1 and 2, the uterine tissues (days 8-10 of the estrous cycle) were collected post mortem from a slaughterhouse in Holon (Israel) from ipsilateral and contralateral horns of the same uterus and divided into two pieces. The first piece was directly frozen and stored in liquid nitrogen for mRNA isolation, and the second one was fixed in $4 \%$ paraformaldehyde (PFA) for immunostaining.

For experiments 3, 4, and 5, the uterine tissues (days 8-10 of the estrous cycle) were collected post mortem at the Meat Processing Plant 'Warmia' (Biskupiec, Poland) and transported on ice to the laboratory within $40 \mathrm{~min}$ for in vitro cultures.

The day of estrous cycle for all experimental cows was estimated by macroscopic observation of the ovaries and uterus (Miyamoto et al. 2000) and by determination of progesterone $\left(\mathrm{P}_{4}\right)$ levels in peripheral blood plasma by direct enzyme immunoassay (EIA).

\section{Uterine cells isolation and in vitro cultures}

The uterine horns ipsilateral to the active ovary, with present corpus luteum, were used for further isolation of cells. The endometrial epithelial and stromal cells were isolated through enzymatic dissociation as described previously (Skarzynski et al. 2000). After endometrial cells isolation, the myometrial layer of the uterus was exposed and then dissected with scissors. About $4 \mathrm{~cm}$ long fragments of muscle tissue were chopped up with scissors into the homogenous material. The chopped tissue ( $\sim \mathrm{g}$ ) was digested in $50 \mathrm{ml}$ of M199 medium (Sigma, M2520) containing $0.1 \%$ of BSA (Sigma, A2058), $20 \mu \mathrm{g} / \mathrm{ml}$ of gentamicin (Sigma, G1271), $2 \mathrm{mg} / \mathrm{ml}$ of collagenase I (Sigma, C0130), $1 \mathrm{mg} / \mathrm{ml}$ of deoxyribonuclease (Sigma, D5025), and $2 \mathrm{mg} / \mathrm{ml}$ of dispase (Life Technologies, 17105041). The enzyme-solution with myometrial tissue was held at $37.5^{\circ} \mathrm{C}$ by stirring for $30 \mathrm{~min}$. After digestion, the cell suspension was filtered through a mesh to remove undigested tissue fragments and then the cells were washed by centrifugation $\left(10 \mathrm{~min}\right.$ at $100 \mathrm{~g}$, at $\left.4{ }^{\circ} \mathrm{C}\right)$. The cells were resuspended in the culture medium (DMEM; Sigma, D5796) supplemented with $10 \%$ of FCS (Sigma, 12133C) and antibiotics (gentamicin/amphotericin B; Life Technologies, 1153727). 
The cells of each layer of the uterus were seeded separately at a density of $1 \times 10^{6}$ of living cells $/ \mathrm{ml}$ in 1 and $2 \mathrm{ml}$ culture medium per well in collagen-coated 24-well and 6-well plates, respectively, or in $8 \mathrm{ml}$ of medium per collagen-coated $25 \mathrm{~cm}^{2}$ flask (Biocoat; BD Bioscience, 4408, 4400, 4484, Bedford, MA, USA) and cultured at $37.5^{\circ} \mathrm{C}$ in a humidified atmosphere of $5 \% \mathrm{CO}_{2}, 95 \%$ air. The medium was changed every 2 days until confluence was reached. Cell culture homogeneity was confirmed using real-time PCR for mRNA expression and determination of VE-cadherin, vimentin, and desmin in epithelial, stromal, and myometrial cells respectively (Zeiler et al. 2007).

For flow cytometry assays, cell cultures from the $25 \mathrm{~cm}^{2}$ flasks, after confluence was reached, were trypsinized with trypsin/EDTA solution (Sigma, T1763). The trypsin solution was washed out by centrifugation $\left(10 \mathrm{~min}\right.$ at $100 \mathrm{~g}$, at $\left.4{ }^{\circ} \mathrm{C}\right)$ with the culture medium containing $10 \%$ of FCS. The cells were resuspended in $1 \mathrm{ml}$ of sterile Hank's Buffered Salt Solution (HBSS; Sigma, H6648) with 1\% of BSA.

\section{Experimental procedure}

Experiment 1: immunodetection of C-KIT and pluripotent markers in bovine uterine tissues

C-KIT and the stem cell markers OCT3/4, NANOG, and SOX2 in different layers of uterine ipsilateral $(n=8)$ and contralateral horns $(n=8)$, i.e., endometrium and myometrium, were localized by immunohistochemistry.

Experiment 2: expression of C-KIT and transcriptional factors OCT3/4, NANOG, and SOX2 mRNA in uterine tissues relative to the age of cows and uterine horn side

This experiment was carried out to assess whether there are changes in C-KIT, OCT3/4, NANOG, and SOX2 gene expression in bovine uterine tissues at different ages of animals and between uterine horns. Cows were divided into two groups: II-IV $(n=12)$ and V-VII lactation $(n=12)$. The level of expression of C-KIT gene indicated the origin of stem cells.

mRNA was isolated from the fragments $(\sim 30 \mathrm{mg})$ of ipsilateral $(n=24)$ and contralateral uterine horns $(n=24)$ consisting of endometrial and myometrial layers. The expression of genes for C-KIT, OCT3/4, NANOG, and SOX2 was determined by quantitative real-time PCR.

Experiment 3: expression of C-KIT, OCT3/4, NANOG, and SOX2 mRNA and protein in uterine cells, and C-KIT receptor ligand levels in uterine cells-conditioned culture media

This experiment was carried out to assess whether there are changes in C-KIT, OCT3/4, NANOG, and SOX2 expression among epithelial, stromal, and myometrial bovine uterine cells $(n=8)$. The level of expression of C-KIT marker was used to indicate the origin of stem cells.

In individual cell cultures, C-KIT, OCT3/4, NANOG, and SOX2 mRNA expression was determined by quantitative real-time PCR. Protein concentrations for pluripotent markers were examined in different cell types by western blotting analysis. The levels of C-KIT receptor ligand, also called stem cell factor (SCF), were measured in the uterine cell culture media by ELISA.

Experiment 4: identification of stem cell markers OCT3/4, NANOG, and SOX2 in different uterine cell populations

This experiment was carried out to determine the presence of pluripotency markers, and the transcriptional factors OCT3/4, $N A N O G$, and $S O X 2$, in cells originating from different layers of the bovine uterus $(n=8)$. For this purpose, cultured epithelial, stromal, and myometrial cell populations were examined by flow cytometry assays (fluorescence-activated cell sorting (FACS)).

Experiment 5: in vitro differentiation of the bovine uterine cells into adipogenic, chondrogenic, and osteogenic lineages

This experiment was carried out to determine the pluripotent/ multipotent properties in the bovine uterine epithelial, stromal, and myometrial cells. Thus, the cells were differentiated in vitro into three mesenchymal lineages under the influence of adipogenic, chondrogenic, and osteogenic media $(n=4)$.

\section{Total RNA isolation}

Total RNA was extracted from uterine tissues and cultured cells using TRI-Reagent (Sigma, T9424) according to the manufacturer's instructions. The content and purity of RNA were assessed on a NanoDrop 1000 (Thermo Fisher Scientific, ND-1000, Wilmington, DE, USA). One microgram of each sample of total RNA was reverse transcribed to cDNA using the QuantiTect Reverse Transcription Kit (Life Technologies, 205313), as described in the supplier's protocol. The cDNA obtained was stored at $-20{ }^{\circ} \mathrm{C}$ until real-time PCR.

\section{Real-time PCR quantification}

Quantitative real-time PCR was performed using the Applied Biosystems 7900 (Applied Biosystems) with the KAPA SYBR FAST qPCR Kit (Kapa Biosystems, KK4602, Boston, MA, USA) according to the manufacturer's instructions. The real-time PCR mix $(20 \mu \mathrm{l})$ contained $19 \mu \mathrm{l}$ of KAPA SYBR FAST Master Mix, $0.5 \mu \mathrm{M}$ of sense and antisense primers, and $1 \mu$ of reverse transcribed cDNA (50 ng). The primer sequences used for determination of C-KIT, OCT3/4, NANOG, SOX2, and glyceraldehyde 3-phosphate dehydrogenase $(G A P D H)$ mRNA expression are given in Table 1. Standard curves consisting of serial dilutions of the appropriate cDNA were plotted for efficiency evaluation. Amplification was preceded by an initial enzyme activation step $\left(3 \mathrm{~min}, 95^{\circ} \mathrm{C}\right)$. The PCR steps were as follows: 40 cycles of denaturation $\left(1 \mathrm{~s}, 95^{\circ} \mathrm{C}\right)$ and then annealing and extending $\left(20 \mathrm{~s}, 60^{\circ} \mathrm{C}\right)$. After amplification, melting curves were acquired by stepwise increases at a temperature of $50-95{ }^{\circ} \mathrm{C}$ to ensure that a single product was amplified and no primer-dimer structures were formed. Control reactions in the absence of the template or primers were performed to confirm that products were free from genomic DNA contamination. Dissociation curves analysis was carried out after each real-time experiment to confirm the presence of only one amplification product. Data were normalized using 
Table 1 Oligonucleotide sequences used for real-time PCR.

\begin{tabular}{|c|c|c|c|}
\hline Gene & Oligonucleotide sequences & Product size (bp) & GenBank \\
\hline GAPDH & $\begin{array}{l}\text { FWD: 5'-CACCCTCAAGATTGTCAGCA-3' } \\
\text { REV: 5'-GGTCATAAGTCCCTCCACGA-3' }\end{array}$ & 103 & BC102589 \\
\hline$C-K I T$ & 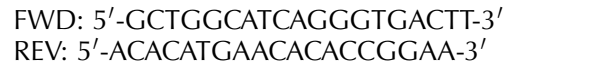 & 110 & NM_001166484.1 \\
\hline OCT3/4 & $\begin{array}{l}\text { FWD: 5'-AGGTGTTCAGCCAAACGACTA-3' } \\
\text { REV: 5'-TCTCCTGCAGATTCTCGTTGT-3 } 3^{\prime}\end{array}$ & 195 & FD381287.1 \\
\hline NANOG & $\begin{array}{l}\text { FWD: 5'-TGCATTTGCTGGAGACTGAG-3' } \\
\text { REV: 55'-GTCCCGGTCAAGAAACAAAA-3' }\end{array}$ & 107 & DQ069776 \\
\hline$S O X 2$ & $\begin{array}{l}\text { FWD: } 5^{\prime} \text {-GCACATGAACGGCTGGAGCAACG-3' } \\
\text { REV: } 5^{\prime} \text {-TGCTGCGAGTAGGACATGCTGTAGG-3' }\end{array}$ & 218 & JQ231229.1 \\
\hline
\end{tabular}

the $\Delta \Delta C_{\mathrm{t}}$ method. The samples were amplified in duplicates. Data are shown as the average fold increase, with S.E.M., and are expressed relative to the housekeeping gene GAPDH.

\section{Fluorescence-activated cell sorting}

In flow cytometry analysis, $1 \mathrm{ml}$ samples of cells were used at a concentration of $1 \times 10^{7} / \mathrm{ml}$. Flow cytometry was performed using a pluripotent stem cell transcription factor analysis kit (BD Stemflow, 560589) according to the manufacturer's procedures. Briefly, single-cell suspensions were washed twice in PBS and fixed in BD cytofix buffer. After washing, cells were resuspended in $1 \times \mathrm{BD}$ perm/wash buffer to permeabilize them. To stain cells, OCT3/4-PerCP Cy5.5, SOX2-Alexa Fluor 647, and NANOG-PE were added for 30 min with incubation at room temperature $\left(21^{\circ} \mathrm{C}\right.$, RT). The antibodies were conjugated with three different fluorochromes and used simultaneously to stain pluripotent transcription factors in the same cell population. Concomitantly, a second sample of the cells was stained with isotype controls (nonspecific binding). Immediately after the cell-stain incubation, flow analysis was performed. All analyses were performed on a BD FACSAria II Cell Sorter (Becton Dickinson, San Jose, CA, USA).

\section{Hormone determination}

$\mathrm{P}_{4}$ levels in plasma were measured using EIA as described previously (Skarzynski et al. 2003). The antibodies (anti-P 4 , code SO/91/4; kindly donated by Prof. S Okrasa, WarmiaMazury University, Olsztyn, Poland) were characterized previously (Ciereszko et al. 2001).

The standard curve ranged from 0.39 to $100 \mathrm{ng} / \mathrm{ml}$ and the effective dose for $50 \%$ inhibition (ED 50) of the assay was $4.5 \mathrm{ng} / \mathrm{ml}$. The intra- and inter-assay coefficients of variation (CV) were 5.5 and $8.5 \%$ respectively.

\section{ELISA}

The SCF were measured using the ELISA Kit (Abcam, 108901, Cambridge, UK) as described in the supplier's protocol. The standard curve ranged from 40 to $0.156 \mathrm{ng} / \mathrm{ml}$ and the minimum detectable dose of C-KIT receptor ligand was $0.15 \mathrm{ng} / \mathrm{ml}$. The intra- and inter-assay coefficients (CV) were 4.5 and $7.2 \%$ respectively.

\section{Immunohistochemistry}

Cross-sections of uterine horn samples were fixed in $4 \%$ PFA in $0.1 \mathrm{M}$ PBS ( $\mathrm{pH} 7.4$ ) and cryoprotected in $18 \%$ sucrose. Immunostaining was carried out on consecutive $7 \mu \mathrm{m}$ cryostat sections. To block endogenous peroxidase, the sections were treated with hydrogen peroxide in methanol and washed in $0.1 \mathrm{M}$ PBS. The sections were blocked with $10 \%$ normal goat serum (Sigma, G9023) for $1 \mathrm{~h}$ at RT, incubated overnight at RT with a 1:100 dilution of anti-C-KIT (Dako, K1906, CA, USA), anti-OCT3/4 (Abcam, 19857), anti-NANOG (Abcam, 80892), or anti-SOX2 (Sigma, S9072) antibodies, washed in PBS, incubated for $1 \mathrm{~h}$ with a 1:25 000 dilution of biotinylated antirabbit (Vectastain ABC Kit; Vector Laboratories, PK 4001, Burlingame, CA, USA) antibodies, then washed, incubated for 45 min with the ABD reagent in PBS, and washed again. Proteins were visualized by incubating the sections in $0.3 \mathrm{mg} / \mathrm{ml}$ 3,30-diaminobenzidine tetrahydrochloride in $0.01 \%$ hydrogen peroxide in Tris-buffered saline $(\mathrm{pH}$ 7.2) for 2-3 min. Finally, the sections were dehydrated and coverslipped with the DPX mounting medium (Park Scientific Ltd, D-11601, Northampton, UK). To determine the specificity of the immunohistochemical staining, two controls were performed: first, the primary antibody was omitted during the
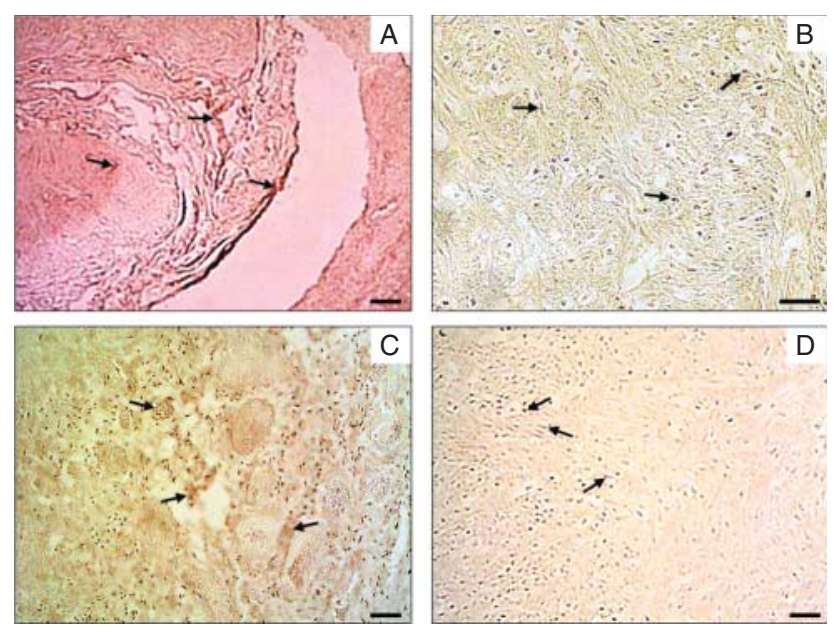

Figure 1 Immunodetection of C-KIT (A) and pluripotency markers OCT3/4 (B), NANOG (C), and SOX2 (D) in bovine uterine tissues. Scale bars: $20 \mu \mathrm{m}$. Arrows indicate the most intense histochemical reactions. 
immunostaining procedure; second, the primary antibody was substituted with a nonspecific IgG. Observations and photographs were made using a light microscope (Nikon FXA, Tokyo, Japan).

\section{Western blotting analysis}

Proteins from in vitro cultured cells were released using a lysis buffer containing $50 \mathrm{mM}$ Tris- $\mathrm{HCl}$ ( $\mathrm{pH} \mathrm{8.0),} 150 \mathrm{mM} \mathrm{NaCl}$, $5 \mathrm{mM}$ EDTA, $0.1 \%$ SDS, 1\% TritonX-100, 0.5\% sodium deoxychelate, and protease inhibitors (Sigma, P8340). The lysates were stored at $-86{ }^{\circ} \mathrm{C}$ until further analysis. Protein concentrations were measured by the Bradford method.

The western blotting analysis was carried out as described previously (Korzekwa et al. 2011). Equal amounts of protein were dissolved in SDS gel-loading buffer, heated to $95^{\circ} \mathrm{C}$ for $4 \mathrm{~min}$, and separated in $8 \%$ SDS-PAGE for C-KIT and 10\% for OCT3/4, NANOG and SOX2. Separated proteins were electroblotted onto $0.2 \mu \mathrm{m}$ nitrocellulose membranes in a transfer buffer. After blocking in 5\% non-fat dry milk in TBS-T buffer for $1.5 \mathrm{~h}$ at RT, the membranes were incubated overnight with a 1:100 dilution of anti-C-KIT (Dako, K1906), anti-OCT3/ 4 (Abcam, 19857), anti-NANOG (Abcam, 80892), or antiSOX2 (Sigma, S9072) antibodies; GAPDH (Sigma, G8795; monoclonal anti-glyceraldehyde-3-phosphate dehydrogenase antibody produced in mouse) expression was used as a reference. Proteins were detected by incubating the membranes with a 1:20 000 dilution of secondary polyclonal antirabbit or anti-goat alkaline phosphatase-conjugated antibodies (Sigma, A 3687, A 3562) for $1.5 \mathrm{~h}$ at RT. The western blots were quantitated using the Kodak $1 \mathrm{D}$ program (Eastman Kodak).

\section{Multilineage differentiation}

Bovine epithelial, stromal, and myometrial cells were cultured separately in standard conditions until $\sim 90 \%$ confluence. Then the cells were stimulated the using Adipogenesis Differentiation Kit (Gibco, Life Technologies, A10070), Chondrogenesis Differentiation Kit (Gibco, Life Technologies, A10071), or Osteogenesis Differentiation Kit (Gibco, Life Technologies, A10072) for 14 days. Differentiating media, supplemented with gentamicin/amphotericin B (Life Technologies, 1153727), were changed every 2-3 days. Simultaneously, for controls, the cells were cultured for 14 days in DMEM (Sigma, D5796) supplemented with 0.1\% BSA and antibiotics (gentamicin/amphotericin B; Life Technologies, 1153727). The cells after adipogenic differentiation and in relative nontreated controls were fixed with $10 \%$ PFA and stained with Oil Red O (Sigma, O0625) to visualize accumulation of lipid droplets. Following chondrogenic differentiation and control cultures, the cells were fixed using 4\% PFA and dyed with Alcian Blue 8GS (AppliChem, A2124, Darmstadt, Germany) to stain glycoproteins and glycosaminoglycans. The cells after osteogenic differentiation and in relative controls were fixed with 4\% PFA and stained with Alizarin Red S (AppliChem, A2290) to visualize calcium deposition (cells mineralization). Observations and photographs were made using an inverted light microscope (Olympus DP25, Tokyo, Japan).
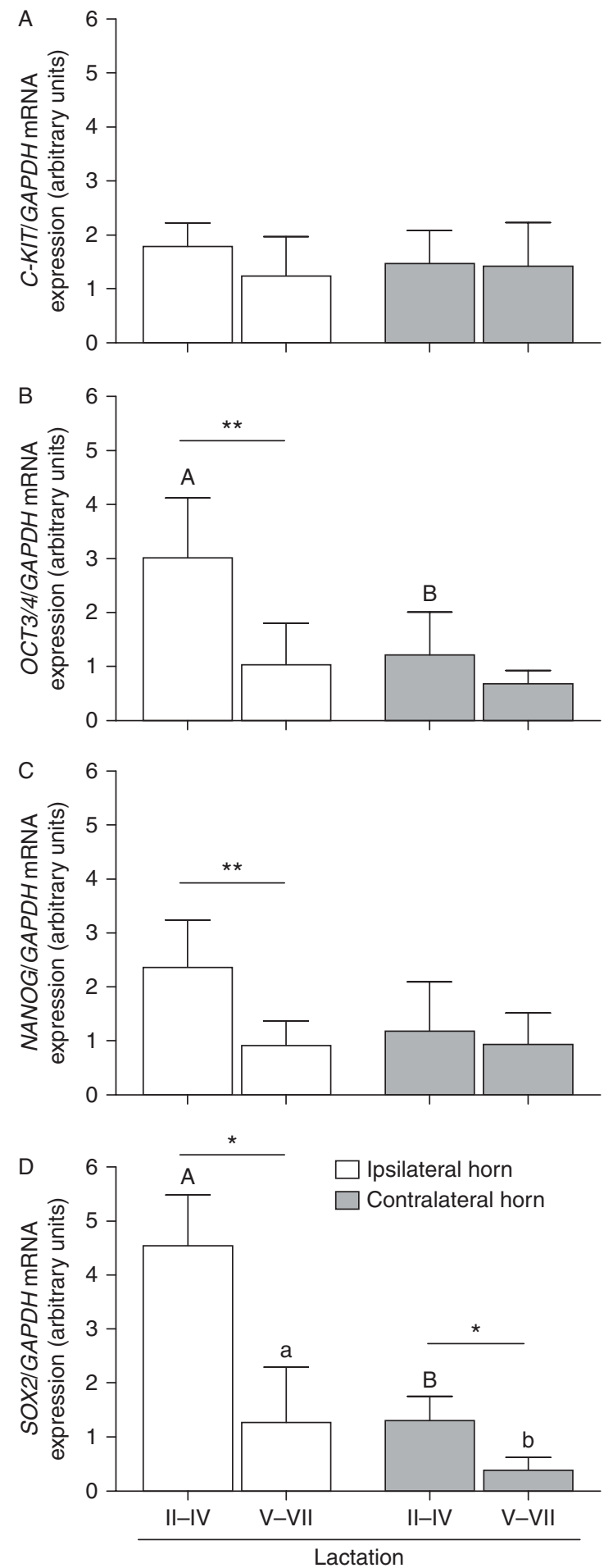

Figure 2 mRNA expression of C-KIT (A), OCT3/4 (B), NANOG (C), and $S O X 2(\mathrm{D})$ in uterine tissues of cows between lactations II-IV and V-VII collected from ipsilateral and contralateral horns. Data were normalized against glyceraldehyde-3-phosphate dehydrogenase (GAPDH). Bars represent the mean \pm S.E.M. Statistical differences between cows in lactations II-IV vs lactations V-VII: ${ }^{*} P<0.05,{ }^{*} P<0.01$, respectively, as determined by Student's $t$-test; statistical differences between ipsilateral and contralateral horns of cow uteri $(P<0.05)$ are indicated by different letters (a, b, A, B), as determined by Student's $t$-test. 


\section{Statistical analyses}

For experiment 2, statistical analysis was performed using Student's $t$-test (GraphPad PRISM Version 5.00, San Diego, CA, USA). All data are expressed as means \pm s.E.M. Differences were analyzed between the ipsilateral and contralateral horn of the same uterus and considered to be significant at $P<0.05$. The differences between bovine uteri collected from younger (II-IV lactation) and older cows (V-VII lactation) were considered to be significant at $* P<0.05$. and $* * P<0.01$.

The statistical significance of differences between epithelial, stromal, and myometrial cells (experiments 3 and 4) was analyzed by one-way ANOVA followed by Newman-Keuls's post hoc test (ANOVA; GraphPAD PRISM), if the initial ANOVA was significant $(P<0.05)$, differences are indicated by letters. All data are expressed as means \pm S.E.M.

\section{Results}

\section{Experiment 1: immunodetection of C-KIT and pluripotent markers in bovine uterine tissues}

Immunohistochemical staining revealed C-KIT localization in the lymphatic and blood vessels of stromal and myometrial endothelium in both horns of the uterus (Fig. 1A). Staining for OCT3/4 was weakly observed mainly in the stromal layer (Fig. 1B), whereas NANOG immunostaining was highly expressed evenly in the stromal layer of both uterine horns (Fig. 1C). SOX2 immunostaining was revealed mainly in the myometrial layer, in the single muscle cells (Fig. 1D) of both uterine horns.

\section{Experiment 2: expression of C-KIT and transcriptional factors OCT3/4, NANOG, and SOX2 mRNA in uterine tissues relative to the age of cows and uterine horn side}

There were no changes in C-KIT mRNA expression either in younger or older cows, or between the ipsilateral and contralateral horns of uteri (Fig. 2A).

Expression of OCT3/4 and NANOG mRNA was upregulated in uterine tissue from the ipsilateral horn in cows between lactations II and IV compared with older cows (between lactations $\mathrm{V}$ and VII, $P<0.01$; Fig. 2B and C). There were no changes in OCT3/4 and NANOG mRNA expression between younger and older cows in the contralateral uterine horns (Fig. 2B and C). OCT3/4 and NANOG mRNA expression was similar in younger or older cows in both uterine horns (Fig. 2B and C), with the exception of OCT3/4 mRNA expression in ipsilateral horns in younger cows, which was higher compared with contralateral horns of younger cows $(P<0.05$; Fig. 2B).

Transcripts for SOX2 in endometrial tissue increased with age. In tissues collected from both ipsilateral and contralateral uterine horns, SOX2 mRNA expression was elevated in cows between lactations II and IV compared with older animals between lactations $\mathrm{V}$ and VII. The expression of SOX2 was higher in ipsilateral than in contralateral uterine horn $(P<0.05$; Fig. 2D).
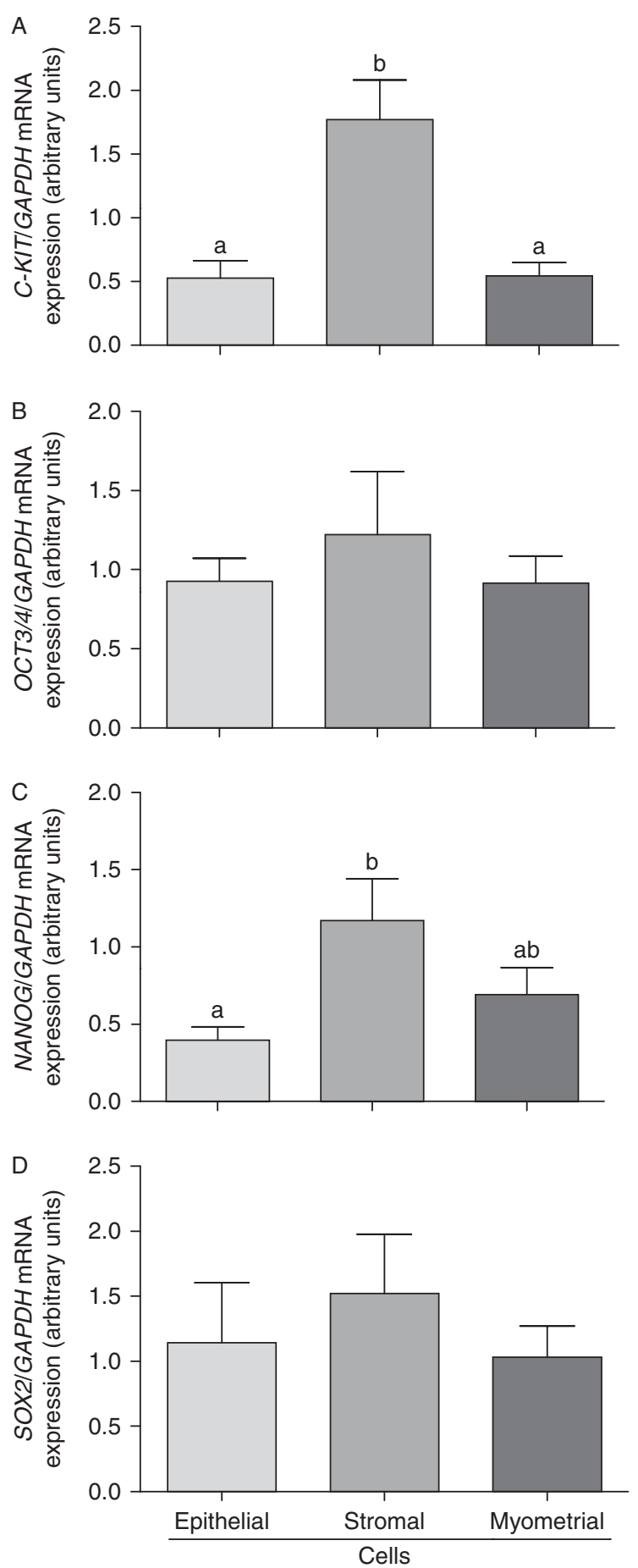

Figure 3 mRNA expression of C-KIT (A), OCT3/4 (B), NANOG (C), and SOX2 (D) in bovine uterine epithelial, stromal, and myometrial cells. Data were normalized against glyceraldehyde-3-phosphate dehydrogenase $(G A P D H)$. Bars represent the mean \pm s.E.M. Statistical differences between uterine cell types $(P<0.05)$ are indicated by different letters $(a, b)$, as determined by one-way ANOVA followed by Newman-Keuls's post hoc test. 
Experiment 3: expression of C-KIT, OCT3/4, NANOG, and SOX2 $\mathrm{mRNA}$ and protein in uterine cells, and C-KIT receptor ligand levels in uterine cell culture media

The cells viability, about $95 \%$ of live cells, was determined by $0.3 \%$ trypan blue dye exclusion.

$C-K I T$ mRNA expression was the highest in stromal cells compared with other uterine cells $(P<0.05$; Fig. 3A). Bovine bone marrow tissue was used as a positive control for C-KIT mRNA expression (data not shown).

There were no significant differences in OCT3/4 and SOX2 mRNA expression, as determined by realtime PCR, in different in vitro-cultured uterine epithelial, stromal, and myometrial cells (Fig. 3B and D). NANOG mRNA expression was the highest in stromal uterine cells, lower in myometrial cells, and the least in epithelial cells $(P<0.05$; Fig. 3C).

There were no significant differences in C-KIT protein concentrations among different types of uterine cells cultured in vitro (Fig. 4A). Bovine bone marrow tissue was used as a positive control for C-KIT protein expression (data not shown).

The patterns for protein expression of OCT3/4, NANOG, and SOX2 were similar to mRNA expression. OCT3/4 protein expression was the highest in stromal uterine cells compared with the other uterine cells $(P<0.05$; Fig. 4B). NANOG protein concentration was the highest in stromal uterine cells, lower in myometrial cells, and the lowest in epithelial cells ( $P<0.05$; Fig. 4C). SOX2 protein concentrations were at the same level in all populations of in vitro-cultured uterine cells (Fig. 4D).

C-KIT receptor ligand level, measured by ELISA, in epithelial and myometrial uterine cells was over $45 \%$ higher than in stromal cells $(P<0.05$; Fig. 5).

\section{Experiment 4: identification of stem cell markers OCT3/4, NANOG, and SOX2 in different uterine cell populations}

OCT3/4, NANOG, and SOX2 were identified in epithelial, stromal, and myometrial uterine cells by flow cytometry analyses (Fig. 6A and B). There were no statistical differences between OCT3/4 and SOX2 fluorimetric staining $(P>0.05$; Fig. $6 \mathrm{~A})$, but NANOG staining was the highest in stromal cells $(P<0.05$; Fig. $6 \mathrm{~A})$.

\section{Experiment 5: in vitro differentiation of the bovine uterine cells into adipogenic, chondrogenic, and osteogenic lineages}

Bovine uterine cells: epithelial, stromal, and myometrial as well, accumulated lipid droplets, after treatment with adipogenesis differentiation medium (Fig. 7A), not observed in non-treated cultures (Fig. 7B). Chondrogenic differentiation was observed in the cells stimulated with the chondrogenic medium (Fig. 7C), but was absent in
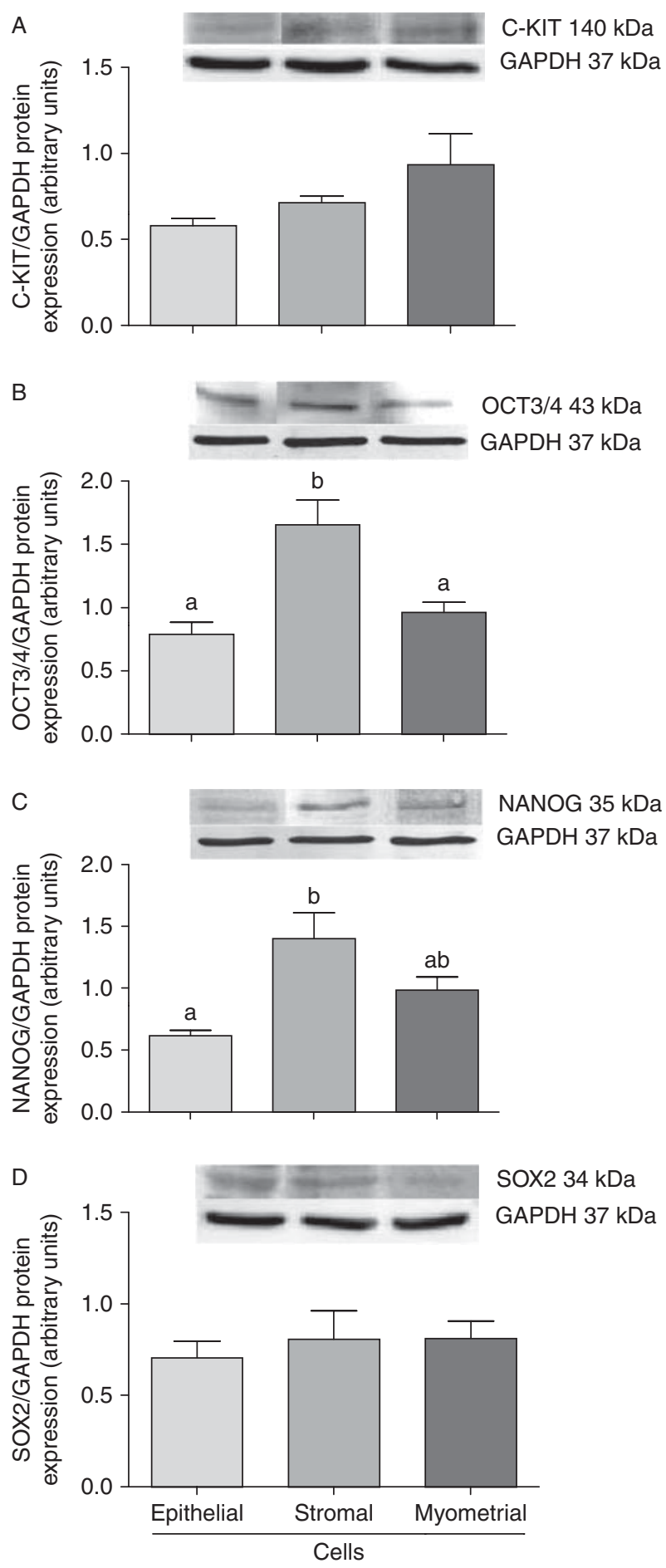

Figure 4 Protein expression of C-KIT (A), OCT3/4 (B), NANOG (C), and SOX2 (D) in bovine uterine epithelial, stromal, and myometrial cells. Data were normalized against glyceraldehyde-3-phosphate dehydrogenase (GAPDH). Bars represent the mean \pm s.E.M. Statistical differences between uterine cell types $(P<0.05)$ are indicated by different letters $(a, b)$, as determined by one-way ANOVA followed by Newman-Keuls's post hoc test. Representative blots for C-KIT, OCT3/4, NANOG, SOX2, and GAPDH are shown above the graphs. 


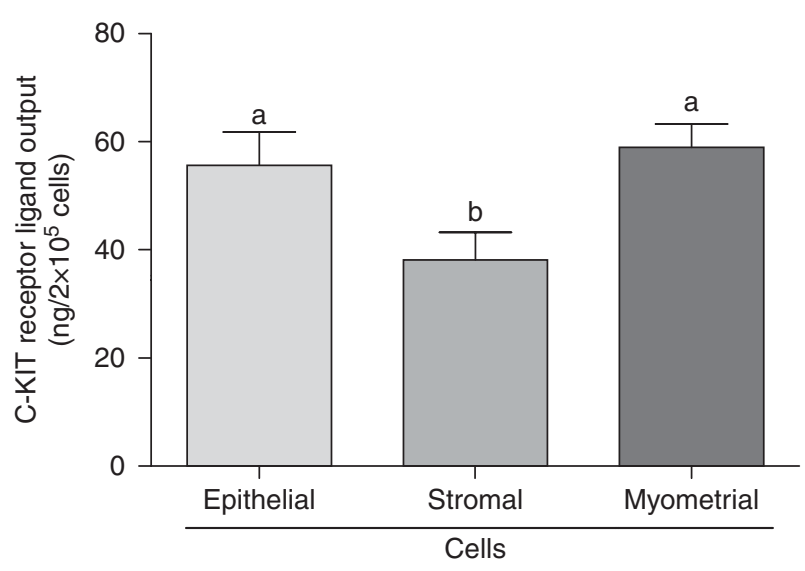

Figure 5 C-KIT receptor ligand concentrations in the culture medium conditioned by bovine uterine epithelial, stromal, and myometrial cells. Bars represent the mean \pm s.E.m. Statistical differences between cell types $(P<0.05)$ are indicated by different letters $(a, b)$, as determined by one-way ANOVA followed by Newman-Keuls's post hoc test.

non-stimulated cells (Fig. 7D). The uterine cells accumulated calcium depots after treatment with osteogenic medium (Fig. 7E), but not in negative control cultures (Fig. 7F).

\section{Discussion}

In this study, we identified pluripotent cells in bovine uterine tissues and in different uterine cell populations. Moreover, we have demonstrated that mRNA expression of stem cell markers in uterine tissues was the highest in the ipsilateral horn compared with the contralateral horn. Furthermore, we examined changes in mRNA expression for pluripotency markers in uterine tissues from cows at lactations II-IV and V-VII, and revealed a decrease in pluripotent transcription factor expression in older cows. We also showed differences in the expression of mRNAs and proteins for the pluripotency markers C-KIT, OCT3/4, NANOG, and SOX2 in in vitrocultured uterine epithelial, stromal, and myometrial cells. Furthermore, we confirmed that the isolated pluripotent/multipotent bovine uterine cells are able to differentiate in vitro under adipogenic, chondrogenic, and osteogenic conditions. To our knowledge, this is the first study that concerned the identification of stem cell markers in the bovine uterus relative to animals' age, uterine horn side, and type of uterine cells.

Donofrio et al. (2008) were the first ones to isolate from the bovine uterus a population of endometrial stromal cells that displayed the capacity to differentiate in vitro into bone tissue. That study suggests that in the bovine endometrium exists a population of cells possessing wide plasticity of phenotype, and that they may differentiate into distinct mesenchymal cell lineages, which are characteristics of MSc (Donofrio et al. 2008). In women, stem cells are present in uterine tissue throughout their whole life (Cho et al. 2004). However, that study focused only on endometrial expression of the hematopoietic stem markers C-KIT and CD34. The expression of C-KIT protein in human endometrium was mainly limited to the basal layer of the uterus and was more abundant in the secretory phase than in the proliferative phase during the female reproductive cycle (Cho et al. 2004). Apart from that report, there is a lack of studies on age-dependent changes in the presence of stem cell markers in the uterus, and there are no such studies in domestic animals. In this study, we showed that all the pluripotent transcription factors were expressed at higher levels in the uteri from cows at lactations II-IV compared with cows at lactations V-VII. These results indicate that stem cells are more abundant in the uteri of younger animals. On the other hand, in both groups C-KIT receptor mRNA expression was at the same level, which suggests that stem cells from bone marrow migrate to the uterus throughout the whole life of the animal and is not agedependent. Moreover, the differences in the mRNA expression of transcription factors SOX2,OCT3/4, and NANOG between ipsilateral and contralateral uterine horns, revealed in this study, indicate a local influence of ovarian steroids on uterine stem cell activity. Whereas mRNA expression of C-KIT at the same level in both horns demonstrates that there is also a population of stem cells in the bovine uterus of medullary origin, they

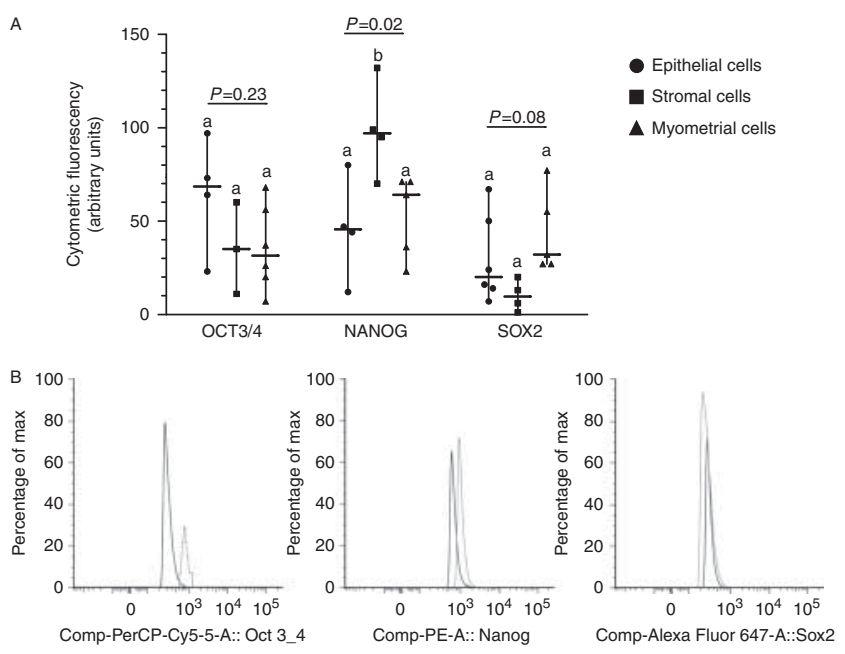

Figure 6 The content of cells positive for stem cell markers (OCT3/4, NANOG, SOX2) in bovine uterine epithelial, stromal, and myometrial cell populations (A). Values represent the differences between medians of the shifts in fluorescence between OCT3/4, NANOG, SOX2, and isotype controls, obtained in flow cytometry assays. Statistical differences between cell types $(P<0.05)$ are indicated by different letters $(a, b)$, as determined by one-way ANOVA followed by NewmanKeuls's post hoc test. Representative histograms with shifts in fluorescence for pluripotent markers (grey histogram) and isotype controls (black histogram; B). 


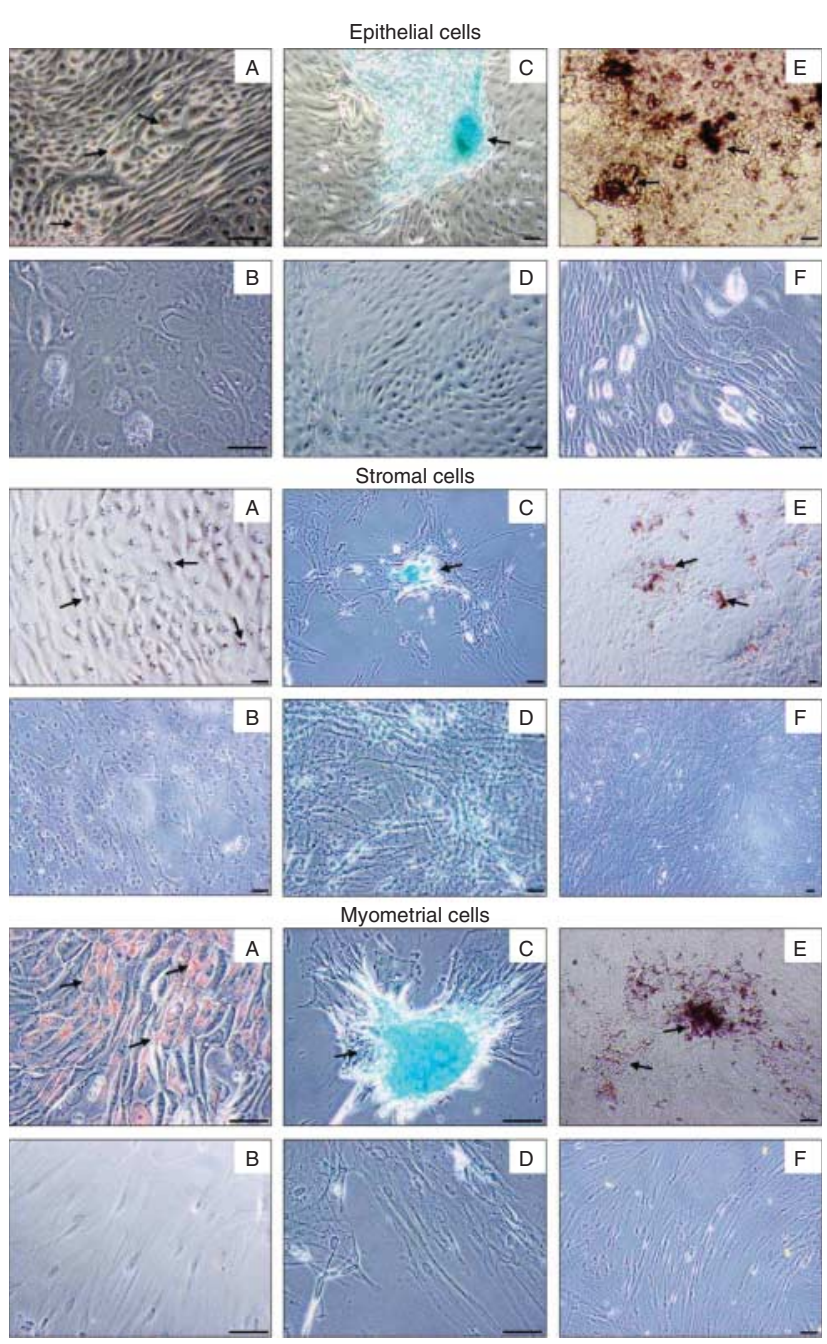

Figure 7 In vitro multilineage differentiation of the bovine uterine cells. Oil Red O staining of the bovine epithelial, stromal, and myometrial cell cultures: lipid droplets accumulated in cytoplasm, dyed in red, visible in cultures treated with adipogenic medium (A), not observed in the controls (B). Alcian Blue 8GS staining of the bovine epithelial, stromal, and myometrial cell cultures: chondrogenic glycoproteins and glycosaminoglycans, stained in blue, observed in cultures stimulated with chondrogenic medium (C), not observed in the controls (D). Alizarin Red S staining of the bovine epithelial, stromal, and myometrial cell cultures: calcium depots, dyed in red, visible in cultures treated with osteogenic medium (E), not observed in the controls (F). Arrows indicate the cells that differentiated.

migrate there independent of the ovarian hormonal status of the animal. Nevertheless, further studies concerning the influence of steroid hormones on bovine stem cell functions in uterus are required.

In this study, we took several approaches to identify stem cells in the populations of bovine uterine cells. Our results clearly confirm that stemness markers such as C-KIT, OCT3/4, NANOG, and SOX2 are expressed not only in cultured endometrial epithelial and stromal cells but also in myometrial uterine cells, at the gene and protein level. These transcription factors indicate the pluripotency of the cells and are expressed in ESc, cancer stem cells, and also in cells residing in reproductive organs as well (Calloni et al. 2013, Luo et al. 2013, Stimpfel et al. 2013). NANOG mRNA expression was elevated in stromal uterine cells in our study. In addition, expression of OCT3/4 and NANOG protein was increased in stromal cells compared with other uterine cells. These results suggest that the endometrial compartment of the uterus is the main site of uterine stem cells in the cow. This is consistent in studies conducted in women, in which stem cells reside mainly in the basal layer of the endometrium, and are responsible for formation of uterine glands and endometrial regeneration (Maruyama et al. 2010). However, in cow, mRNA and protein expression and immunostaining all showed the presence of stem cell markers in the uterus as well as in the myometrial layer. In women's uteri, stem cells have already been identified in the myometrium (Teixeira et al. 2008, Maruyama et al. 2010, 2013, Figueira et al. 2011). In this part of the uterus, even though stem cells play an important role in uterine enlargement during pregnancy, they may also contribute for the development of myometrial cancer in woman (Maruyama et al. 2010) and structural and functional dysfunction, such as adenomyosis, in cows (Korzekwa et al. 2013). We confirmed the presence of pluripotent markers in uterine cells by flow cytometry. We identified a population of cells carrying the stem cell markers OCT3/4, NANOG, and SOX2 in the in vitro-cultured cells isolated from particular uterine layers. Our cytometric results suggest that stem cells constitute a small population of the cells in the bovine uterus. This suggestion is consistent with studies on women, mice, and pigs, in which different stem cell identification approaches showed that in the uterine tissue of these species the pluripotent/multipotent cells populations were minor (Chan et al. 2004, Teixeira et al. 2008, Miernik \& Karasinski 2012). Stem cells also persist in small amounts throughout life in other regenerative adult tissues such as epithelia, bone marrow, and dental pulp (Pittenger et al. 1999, Gronthos et al. 2000, Slack 2000). However, stem cells with their highly proliferative properties even in small populations are able to regenerate and renew tissues in the event of damage, as well as in normal functions (Slack 2000).

We also demonstrated the pluripotency/multipotency of bovine uterine epithelial, stromal, and myometrial cells by multilineage differentiation assay. The uterine cells differentiated in vitro, under proper conditions, into three mesenchymal lineages: adipogenic, chondrogenic, and osteogenic. These results suggest the wide plasticity of phenotype of bovine uterine cells and the presence of undifferentiated stem cells in the bovine uterus, thus we confirmed and extended the results obtained by Donofrio et al. (2008).

The presence of small populations of stem cells in the uterus is evident, but it remains unclear whether these 
cells primarily reside in the uterus or whether they migrate there from bone marrow through blood vessels (Maruyama et al. 2010). It is proven that bone marrowderived stem cells (BMSC) may settle and differentiate in the uterus. After bone marrow transplantation in women and mice, genetic chimerism of endometrial cells was observed (Figueira et al. 2011). Moreover, mouse BMSC under the influence of estradiol $\left(E_{2}\right)$ and the environment of endometrial stromal cells differentiated into endometrial epithelial cells (Zhang et al. 2012). Therefore, bone marrow may also constitute the source of cells involved in endometrial regeneration (Figueira et al. 2011). In our study, we identified pluripotency markers, transcription factors, characteristic of ESc (OCT3/4, NANOG, and SOX2), as well as C-KIT receptor, which is believed to be the typical marker for hematopoietic stem cells derived from bone marrow (Cho et al. 2004). Hence, we presumed that C-KIT is a marker distinguishing BMSC in uterine tissue from residual stem cells. As immunostaining showed, C-KIT receptor was abundantly expressed in cells localized near blood vessels, which may indicate the medullary origin of cells containing C-KIT (Woidacki et al. 2013). Furthermore, C-KIT mRNA expression was increased in cultured bovine uterine stromal cells, which suggests that this compartment of the endometrium is the main location where hematopoietic stem cells settle. Elevated expression of C-KIT mRNA in cultured stromal cells confirms the high proliferative potential of these cells (Woidacki et al. 2013). In the medium conditioned by culture of stromal cells vs epithelial and myometrial cells, decreased levels of SCF, which binds to C-KIT, may suggest activation of signaling through C-KIT by utilizing SCF, which is characteristic for stem cells (Zhang \& Anthony 1994).

We have shown that pluripotent markers are expressed in uterine tissue depending on age and horn side, which suggests the influence of ovarian hormones on uterine stem cells properties. Furthermore, our results indicate that stem cells are present mainly within the bovine uterine stromal cell population but also among epithelial and myometrial cell populations. Thus, uterine stem cells may play an important role in bovine reproduction, and resolution of this issue will require further studies.

\section{Declaration of interest}

The authors declare that there is no conflict of interest that could be perceived as prejudicing the impartiality of the research reported.

\section{Funding}

This work was supported by Grant-in-Aid for Scientific Research from the National Science Centre (2011/01/B/NZ5/ 00939).

\section{Acknowledgements}

The authors thank W Krzywiec for technical support and tissue collection and E Piotrowicz for assistance during experiments. They also thank Prof. S Okrasa of the University of Warmia and Mazury in Olsztyn (Poland) for $\mathrm{P}_{4}$ antiserum, and $M$ Domin - the owner of the slaughterhouse (Meat Processing Plant 'Warmia', Biskupiec, Poland) for permitting collection of the material.

\section{References}

Calloni R, Cordero EA, Henriques JA \& Bonatto D 2013 Reviewing and updating the major molecular markers for stem cells. Stem Cells and Development 22 1455-1476. (doi:10.1089/scd.2012.0637)

Chan RW, Schwab KE \& Gargett CE 2004 Clonogenicity of human endometrial epithelial and stromal cells. Biology of Reproduction $\mathbf{7 0}$ 1738-1750. (doi:10.1095/biolreprod.103.024109)

Cho NH, Park YK, Kim YT, Yang H \& Kim SK 2004 Lifetime expression of stem cell markers in the uterine endometrium. Fertility and Sterility $\mathbf{8 1}$ 403-407. (doi:10.1016/j.fertnstert.2003.07.015)

Ciereszko R, Opałka M, Kamińska B, Wojtczak M, Okrasa S \& Dusza L 2001 Luteotrophic action of prolactin during the early luteal phase in pigs: the involvement of protein kinases and phosphatases. Reproductive Biology 1 62-83.

Donofrio G, Franceschi V, Capocefalo A, Cavirani S \& Sheldon IM 2008 Bovine endometrial stromal cells display osteogenic properties. Reproductive Biology and Endocrinology 65 1-9. (doi:10.1186/14777827-6-65)

Du H \& Taylor HS 2009 Stem cells in human reproduction. Reproductive Sciences 16 126-139. (doi:10.1177/1933719108329956)

Figueira PG, Abrão MS, Krikun G \& Tylor H 2011 Stem cells in endometrium and their role in the pathogenesis of endometriosis. Annals of the New York Academy of Sciences 1221 10-17. (doi:10.1111/j.17496632.2011.05969.x)

Gargett CE 2004 Stem cells in gynaecology. Australian and New Zealand Journal of Obstetrics and Gynaecology 44 380-386. (doi:10.1111/ j.1479-828X.2004.00290.x)

Gargett CE, Nguyen HPT \& Ye L 2012 Endometrial regeneration and endometrial stem/progenitor cells. Reviews in Endocrine \& Metabolic Disorders 13 235-251. (doi:10.1007/s11154-012-9221-9)

Gronthos S, Mankani M, Brahim J, Robey PG \& Shi S 2000 Postnatal human dental pulp stem cells (DPSCs) in vitro and in vivo. PNAS 97 13625-13630. (doi:10.1073/pnas.240309797)

Izadpanah R, Joswig T, Tsien F, Dufour J, Kirijan JC \& Bunnell BA 2005 Characterization of multipotent mesenchymal stem cells from the bone marrow of rhesus macaques. Stem Cells and Development 14 440-451. (doi:10.1089/scd.2005.14.440)

Korzekwa AJ, Bodek G, Bukowska J, Blitek A \& Skarzynski DJ 2011 Characterization of bovine immortalized luteal endothelial cells: action of cytokines on production and content of arachidonic acid metabolites. Reproductive Biology and Endocrinology 9 1-9. (doi:10.1186/14777827-9-27)

Korzekwa AJ, Bah MM, Gęstwicka M, Socha B \& Skarżyński DJ 2013 Adenomyosis in the bovine uterus: correlation between frequency, age, and 17ß-estradiol-progesterone equilibrium. Theriogenology $\mathbf{7 9}$ 165-172. (doi:10.1016/j.theriogenology.2012.09.023)

Lee KS, Nah JJ, Lee BC, Lee HT, Lee HS, So BJ \& Cha SH 2013 Maintenance and characterization of multipotent mesenchymal stem cells isolated from canine umbilical cord matrix by collagenase digestion. Research in Veterinary Science 94 144-151. (doi:10.1016/j.rvsc.2012.07.033)

Luo W, Li S, Peng B, Ye Y, Deng X \& Yao K 2013 Embryonic stem cells markers Sox2, Oct4 and Nanog expression and their correlations with epithelial-mesenchymal transition in nasopharyngeal carcinoma. PLOS ONE 8 e56324. (doi:10.1371/journal.pone. 0056324)

Maruyama T, Masuda H, Ono M, Kajitani T \& Yoshimura Y 2010 Human uterine stem/progenitor cells: their possible role in uterine physiology and pathology. Reproduction 140 11-22. (doi:10.1530/REP09-0438) 
Maruyama T, Ono M \& Yoshimura Y 2013 Somatic stem cells in the myometrium and in myomas. Seminars in Reproductive Medicine 31 77-81. (doi:10.1055/s-0032-1331801)

Miernik K \& Karasinski J 2012 Porcine uterus contains a population of mesenchymal stem cells. Reproduction 143 203-209. (doi:10.1530/REP11-0202)

Miyamoto Y, Skarzynski DJ \& Okuda K 2000 Is tumor necrosis factor $\alpha$ a trigger for the initiation of endometrial prostaglandin $F_{2 \alpha}$ release at luteolysis in cattle? Biology of Reproduction 62 1109-1115. (doi:10. 1095/biolreprod62.5.1109)

Ono M, Qiang W, Serna VA, Yin P, Coon V JS, Navarro A, Monsivais D, Kakinuma T, Dyson M, Druschitz S et al. 2012 Role of stem cells in human uterine leiomyoma growth. PLoS ONE 7 e36935. (doi:10.1371/ journal.pone.0036935)

Pittenger MF, Mackay AM, Beck SC, Jaiswal RK, Douglas R, Mosca JD, Moorman MA, Simonetti DW, Craig S \& Marshak DR 1999 Multilineage potential of adult human mesenchymal stem cells. Science $\mathbf{2 8 4}$ 143-147. (doi:10.1126/science.284.5411.143)

Schwab KE \& Gargett CE 2007 Co-expression of two perivascular cell markers isolates mesenchymal stem-like cells from human endometrium. Human Reproduction 22 2903-2911. (doi:10.1093/humrep/dem265)

Schwab KE, Hutchinson P \& Gargett CE 2008 Identification of surface markers for prospective isolation of human endometrial stromal colonyforming cells. Human Reproduction 23 934-943. (doi:10.1093/humrep/ den051)

Skarzynski DJ, Miyamoto Y \& Okuda K 2000 Production of prostaglandin $\mathrm{F}_{2 \alpha}$ by cultured bovine endometrial cells in response to tumor necrosis factor $\alpha$ : cell type specificity and intracellular mechanisms. Biology of Reproduction 62 1116-1120. (doi:10.1095/biolreprod62.5.1116)

Skarzynski DJ, Bah MM, Deptula KM, Woclawek-Potocka I, Korzekwa A, Shibaya M, Pilawski W \& Okuda K 2003 Roles of tumor necrosis factor- $\alpha$ of the estrous cycle in cattle: an in vivo study. Biology of Reproduction 69 1907-1913. (doi:10.1095/biolreprod.103.016212)

Slack JM 2000 Stem cells in epithelial tissues. Science 287 1431-1433. (doi:10.1126/science.287.5457.1431)

Spencer TE, Johnson GA, Burghardt RC \& Bazer FW 2004 Progesterone and placental hormone actions on the uterus: insights from domestic animals. Biology of Reproduction 71 2-10. (doi:10.1095/biolreprod.103.024133)
Stimpfel M, Skutella T, Cvjeticanin B, Meznaric M, Dovc P, Novakovic S, Cerkovnik P, Vrtacnik-Bokal E \& Virant-Klun I 2013 Isolation, characterization and differentiation of cells expressing pluripotent/multipotent markers from adult human ovaries. Cell and Tissue Research 354 593-607. (doi:10.1007/s00441-013-1677-8)

Teixeira J, Rueda BR \& Pru JK 2008 Uterine stem cells. In StemBook, ed. The Stem Cell Research Community, Cambridge, MA: Harvard Stem Cell Institute. (doi:10.3824/stembook.1.16.1)

Woidacki K, Popovic M, Metz M, Schumacher A, Linzke N, Teles A, Poirier F, Fest S, Jensen F, Rabinovich GA et al. 2013 Mast cells rescue implantation defects caused by c-kit deficiency. Cell Death \& Disease $\mathbf{4}$ e462. (doi:10.1038/cddis.2012.214)

Xu J, Hu FF, Cui YG, Luo J, Jiang CY, Gao L, Qian XQ, Mao YD \& Liu JY 2011 Effect of estradiol on proliferation and differentiation of side population stem/progenitor cells from murine endometrium. Reproductive Biology and Endocrinology 9 103. (doi:10.1186/14777827-9-103)

Zeiler M, Leiser R, Johnson GA, Tinneberg HR \& Pfarrer C 2007 Development of an in vitro model for bovine placentation: a comparison of the in vivo and in vitro expression of integrins and components of extracellular matrix in bovine placental cells. Cells, Tissues, Organs 186 229-242. (doi:10.1159/000107947)

Zhang Z \& Anthony RV 1994 Porcine stem cell factor/c-kit ligand: its molecular cloning and localization within the uterus. Biology of Reproduction 50 95-102. (doi:10.1095/biolreprod50.1.95)

Zhang WB, Cheng MJ, Huang YT, Jiang W, Cong Q, Zheng YF \& Xu CJ 2012 A study in vitro on differentiation of bone marrow mesenchymal stem cells into endometrial epithelial stem cells in mice. European Journal of Obstetrics, Gynecology, and Reproductive Biology 160 185-190. (doi:10.1016/j.ejogrb.2011.10.012)

Received 10 July 2014

First decision 12 November 2014

Revised manuscript received 17 December 2014

Accepted 2 January 2015 